\title{
Percutaneous treatment of congenitally pulmonary vein stenosis in adult patient: $A$ case report
}

Congenital pulmonary vein stenosis (PVs) is an infrequent and poor prognosis anomaly due to associated cardiac anomaly and progressive pulmonary hypertension. In this report we described congenitally PVs without associated anomaly in adult patient that successfully treated percutaneously.

Keywords: Congenital pulmonary vein stenosis - Percutaneously

\section{Introduction}

Congenital pulmonary vein stenosis (PVs) is an infrequent anomaly that occurs in nearly $0.03-0.4 \%$ of patients with congenital heart disease, and commonly diagnosed throughout the neonatal period [1] and with regard to progressive pulmonary hypertension or associated cardiac anomalies survival to adulthood is very unusual in this situation. General mortality rate of this anomaly has been reported to be $48-49 \%$ [2].

Partial or complete abolition of the PVs on one or both sides is a special anomaly due to failed incorporation of the common PV into the left atrium (LA) in the fetal heart development [3]. This anomaly is usually diagnosed in the first 3 years of life, but it may be hindered till maturity in some patients [4].

In this paper, we described very rare case of congenital PVs, that survived to adulthood and successfully treated percutaneously.

\section{Case Report}

A 36 years old woman with complaint from progressive dyspnea from 5 years ago that exacerbated recently arrived to our hospital with respiratory distress and cough and orthopnea and PND. The patient had been healthy but with history of recurrent admission for pneumonia and had no significant personal or family history. She was admitted with diagnosed pulmonary edema but she was not response to medical treatment. Physical examination showed tachycardia and fine crackle in both lungs from base to apex. There was no obvious cardiac murmur. EKG showed sinus tachycardia and non-specific ST-T change (Figure 1).

The chest X-ray showed signs of congestion (peri-broncho vascular and septal thickening, Kerlley B lines, alveolar edema) and a blunted right costo-phrenic angle in favor of pulmonary edema (Figure 2). Transthoracic two-dimensional Doppler echocardiography showed continuous turbulent flow in the small sized LA. Severe right ventricle enlargement and dysfunction and relatively small left ventricle (LVEDVI: $35 \mathrm{cc} / \mathrm{m}^{2}$ ) with moderate dysfunction (EF: 40\%) and sever PAH. The following trans esophageal echocardiography 3D (TEE-3dimentional) revealed LAA appendage compressed with dilated confluent left pulmonary vein and has turbulent flow with LAA velocity: $67 \mathrm{~cm} / \mathrm{s}$ ).

Drainage of left upper PV are confluent with narrow ostium $(3 \mathrm{~mm})$ and turbulent flow
Zahra Khajali, Ata Firoozi, Mozhgan Parsaei and Maryam Aliramezany*

Rajaie Cardiovascular, Medical and Research Center, Iran University of Medical Sciences, Tehran, Iran

*Author for correspondence: Email: maliramezany@yahoo.com Received date: January 24, 2019 Accepted date: March 20, 2019 Publication date: March 25, 2019 


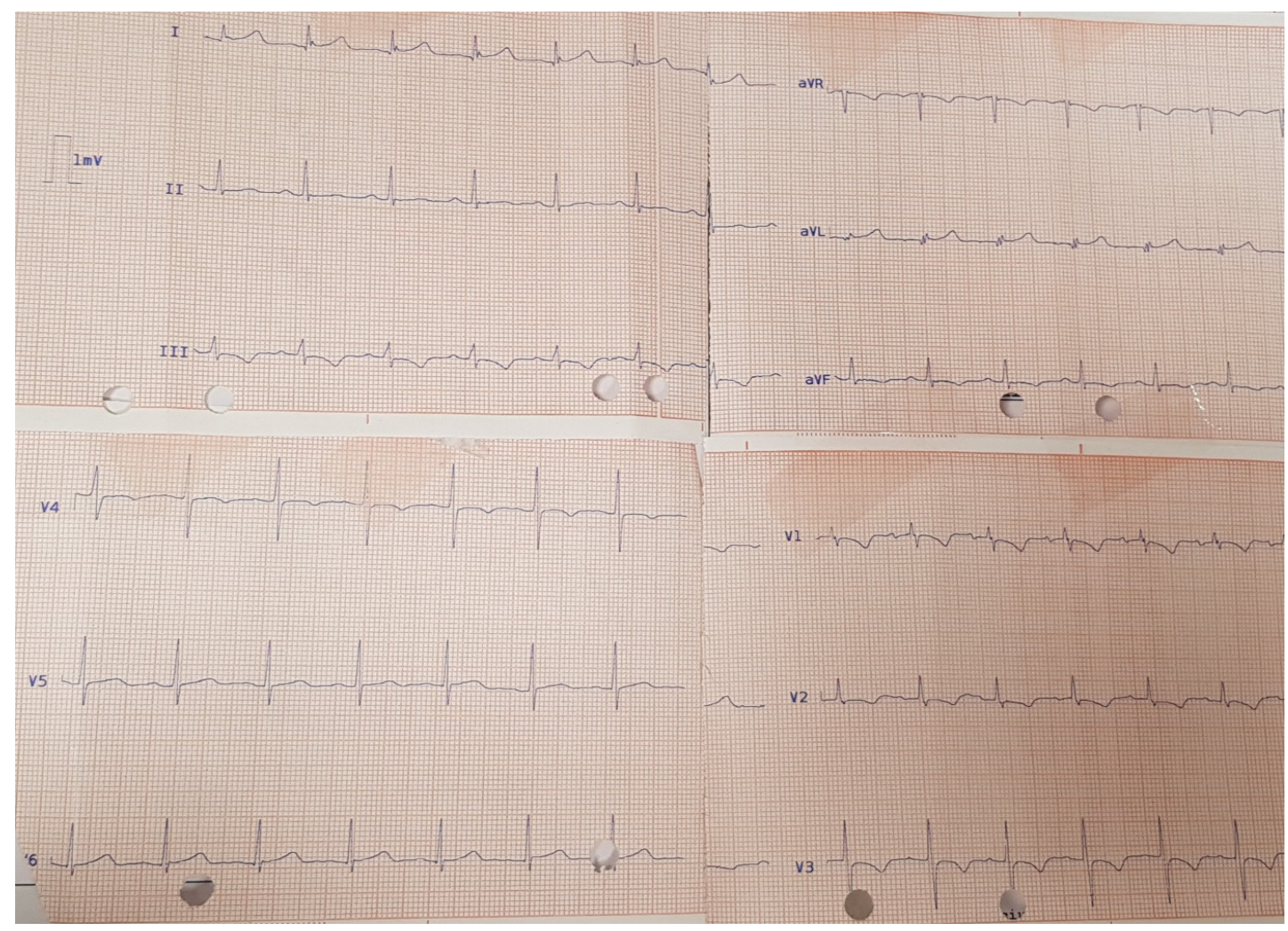

Figure 1: EKG showed sinus tachycardia and non-specific ST-t change.

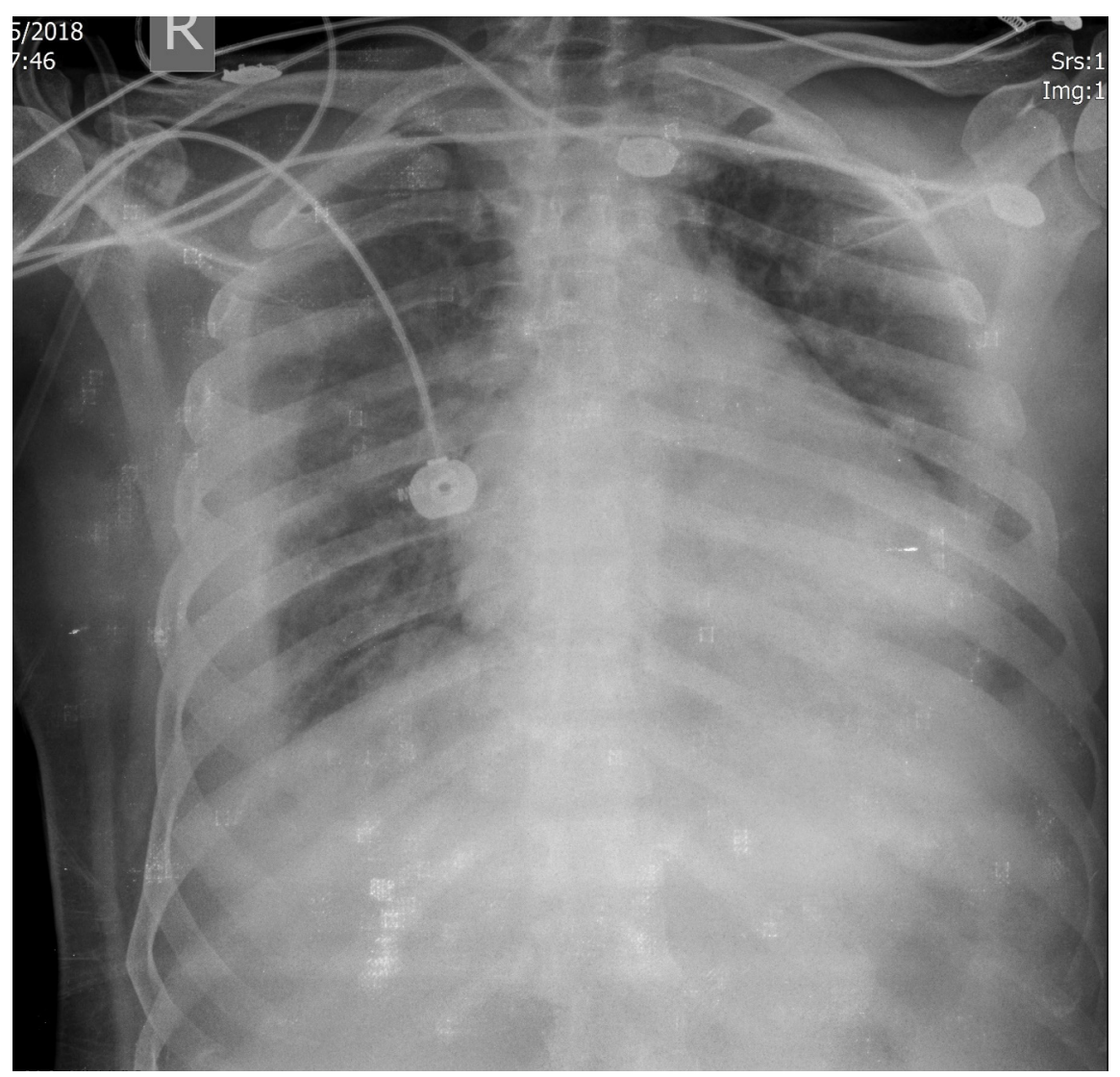

Figure 2: CXR showed sign of pulmonary congestion. 
(MG: $26 \mathrm{mmHg})$ to LA prestenotic dilation $(1.5 \times 1.5)$. Drainage of right upper PV is turbulent to LA with increased gradient $(22 \mathrm{mmHg})$ and drainage of other PV not good seed with TEE. Mild to moderate TR and PI with TRG: $70 \mathrm{mmHg}$ was detected and no other cardiac anomaly was seen (Figure 3).

For better evaluation of all PVs and associated anomaly, pulmonary artery CT angiography (with dynamic contrast medium) was performed and revealed:

1. Right upper lobe PV stenosis at the junction to LA with web formation

2. Stenosis at junction of left common pulmonary vein trunk to LA

3. Enlargement of main PA and branches' in favor of $\mathrm{PAH}$ with increased thickening and congestion on both lungs.

For confirmation of diagnosis and probability of treatment approach, right heart catheterization was performed. The pulmonary artery systolic/diastolic pressure was $100 / 60 \mathrm{mmHg}$ and a pulmonary capillary wedge pressure of $40 \mathrm{mmHg}$ on both sides with increased PVR (18 Wood).

The patient was finally diagnosed with congenital PVs and with regards of symptoms and significant gradient in PV, balloon angioplasty of PVs was performed. After prep and drep, via right femoral vein, sept ostomy performed and then a 0.014 in. guide wire was advanced to RUPV and secondly LUPV and balloon veno- plasty was done by POWERFLEX PRO PTA dilation catheter (OWT) $12812 \mathrm{~mm}$, MAVERICK2.5×15 mm and CRISTAL balloon CBP8X3035/110, EXTREME $8 \times 20 \mathrm{~mm}$ and BIB (balloon in balloon) $8 \times 30 \mathrm{~mm}$ with acceptable final result (Figure 4).

After the procedure, the web-like structure was visible through TEE, but reduction of peak/mean pressure gradient to $15 / 5 \mathrm{mmHg}$ was seen. The patient was discharged 3 days after the procedure without complication. Transthoracic echocardiography performed after 3 and 6 months and 1 year after the PV angioplasty still revealed flow acceleration in the LA but the peak/mean pressure gradient via the stenotic PV was $8 / 6 \mathrm{mmHg}$ but patient has not any symptom during routine follow-up.

\section{Discussion}

Stenosis in pulmonary vein in adult or child patients without any obvious previous or simultaneous source, is named "congenital" PVs that has various form with distinct shelf, narrowing of long segment at the junction of the pulmonary vein and left atrium, or as diffuse hypoplasia of the pulmonary veins [5].

This anomaly is a rare Couse of obstruction of blood flow between pulmonary vein and left atrium that may be involve the adjacent segment of extra pulmonary vein with high mortality rate [2]. For the reason that frequent associated anomaly (about 30-80\% (5)) (secundum type atrial septal defect, anomaly of caval vein, atrioventricular septal defect) or extra cardiac

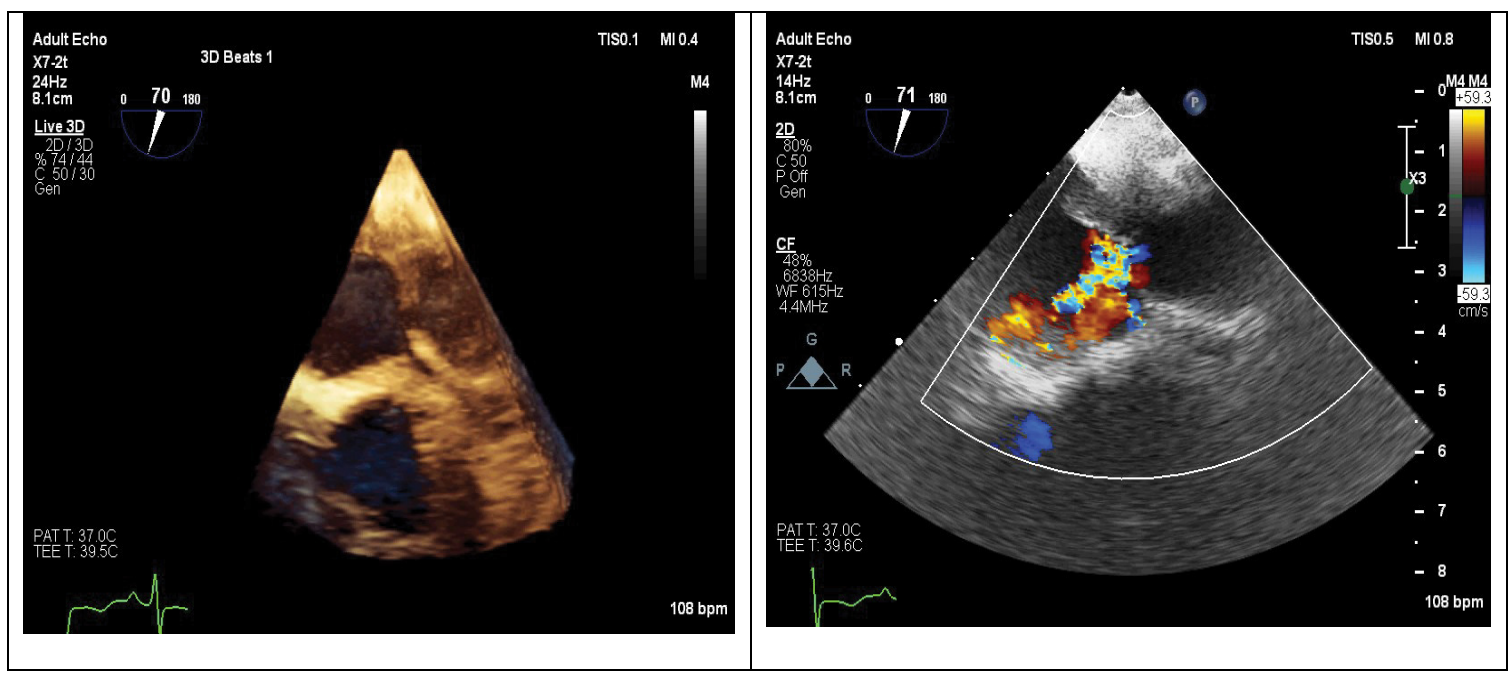

Figure 3: Transesophasial echocardiography (2D-3D) at PV level showed abnormal turbulent flow in LUPV with web like stenosis and post stenotic dilation. 
anomalies (Down, Smith-Lemli-Opitz syndrome) and progressive increased of pulmonary hypertension [6]. As regards to high rate of associated anomaly in this patients, careful imaging protocol for evaluation is recommended [4] number of involved pulmonary vein and degree of obstruction, is determinant of the onset and severity of sign and symptoms patients with pulmonary vein stenosis [5]. The patient in this report was diagnosed with isolated PVs and had no other cardiac or extra cardiac anomalies.

Many researched clarified that any chronic stress on cardiac chamber or great vessels or pulmonary veins may have evoked multiple changes in some membrane channel structures that influence on signal propagation (electrical and chemical) between adjacent myocytes. Another evidence suggests that gap junction remodeling leads to slowed action potential propagation that promotes the development of cardiac arrhythmias, most likely due to its important role in stabilizing and maintaining reentry arrhythmias [7]. Stress-activated
c-Jun N-terminal kinase (JNK) signaling contributes to AF development was seen in different situation for example aging, heart failure and alcohol drinking and prompted $\mathrm{AF}$ and increased cause of mortality and morbidity in patients [8]. For this reason, correction of reversible Couse of cardiac stress is necessary in any age.

Several surgical methods were performed for treatment of this anomaly including scar elimination, primary repair, stent placement during operation, or suture-less pericardial marsupialization but percutaneous trans catheter balloon angioplasty has been newly used and may be an excellent therapeutic choice for isolated PVs [2]. However, there is no agreement about the optimum approach for treatment of this anomaly [9-10].

\section{Conclusion}

In this study we reported a patient with PVs who stayed alive adulthood and treated with percutaneous balloon angioplasty successfully. In our point of view, the fact that the lesion of the current patient was not diffused

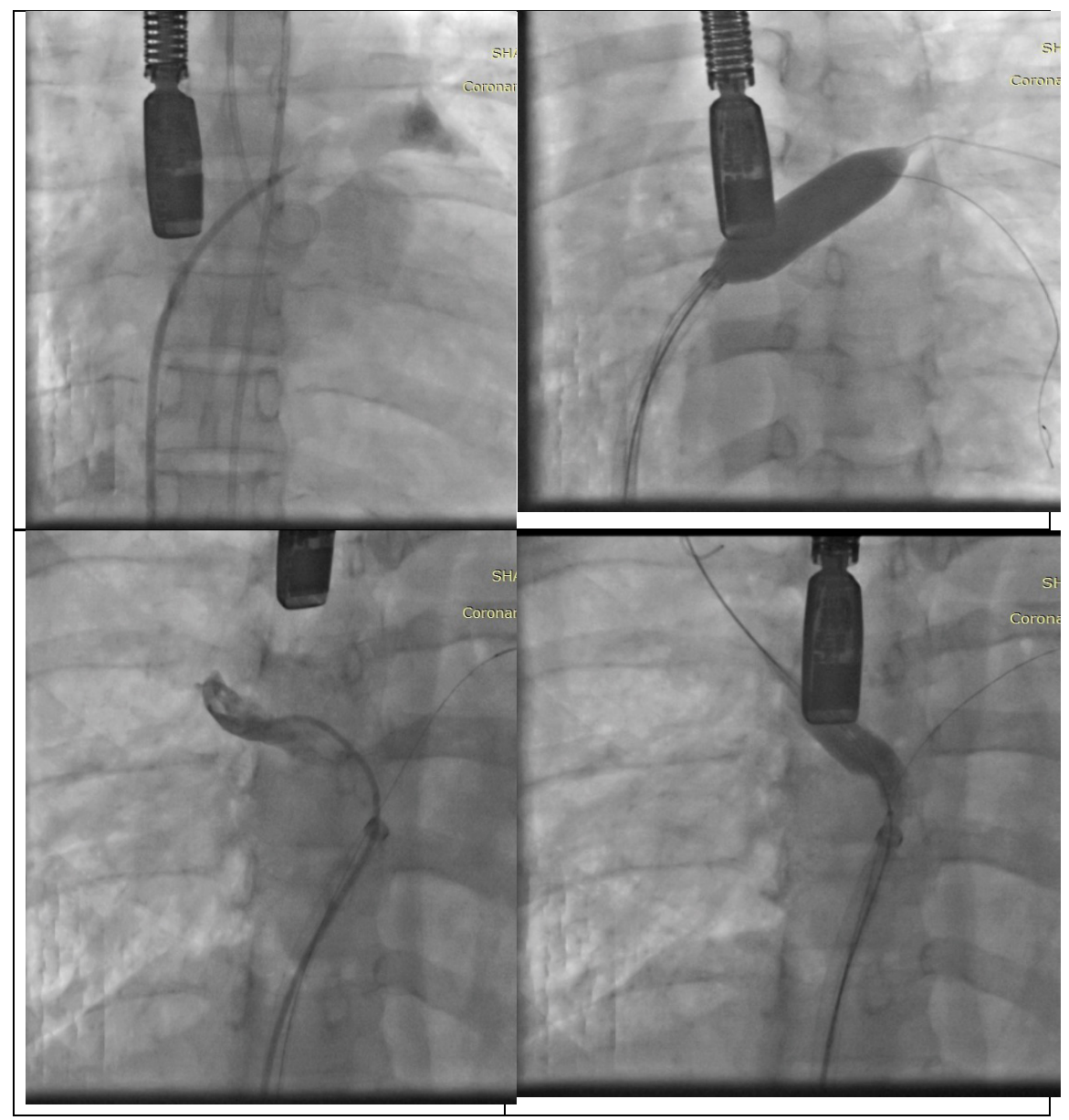

Figure 4: Angiography at AP view showed balloon angioplasty of LUPV and RUPV with good result. 
but instead, was a simple membranous, caused a benign clinical course. Additionally, absence of associated anomaly contributed to the good prognosis.

Most of patients with PVs (either congenital or acquired, relapse of stenosis occurs but percutaneous

\section{References}

1. Holt DB, Moller JH, Larson S, et al. Primary pulmonary vein stenosis. Am J Cardiol. 99(4): 568-72 (2007).

2. Kim YH, Pak HN, Park SM, et al. Congenital pulmonary vein stenosis in an adult patient treated with transcatheter balloon angioplasty. Eur J Echocardiogr. 12(3): E13 (2010).

3. Porres DV, Morenza ÓP, Pallisa E, et al. Learning from the pulmonary veins. Radiographics. 33(4): 999-1022 (2013).

4. Pazos-López P, García-Rodríguez C, Guitián-González A, et al. Pulmonary vein stenosis: etiology, diagnosis and management. World J Cardiol. 8(1): 81 (2016).

5. Latson LA, Prieto LR. Conge nital and acquired pulmonary vein stenosis. Circulation. 115(1): 103-8 (2007).

6. Seale AN, Webber SA, Uemura H, et al. Pulmonary vein stenosis: the UK, Ireland and sweden collaborative study. Heart (2009). balloon angioplasty is a rational therapeutic option for treatment of congenital PVs in adult. We recommended that patient must then be followed carefully with noninvasive imaging test.

7. Yan J, Thomson JK, Zhao W, et al. The stress kinase JNK regulates gap junction $\mathrm{Cx} 43$ gene expression and promotes atrial fibrillation in the aged heart. J Mol Cell Cardiol. 114: 105-15 (2018).

8. Yan J, Thomson JK, Zhao W, et al. Role of stress kinase JNK in binge alcohol-evoked atrial arrhythmia. J Am Coll Cardiol. 71(13): 1459-70 (2018).

9. Devaney EJ, Chang AC, Ohye RG, et al. Management of congenital and acquired pulmonary vein stenosis. Ann Thorac Surg. 81(3): 992-6 (2006).

10. Peng LF, Lock JE, Nugent AW, et al. Comparison of conventional and cutting balloon angioplasty for congenital and postoperative pulmonary vein stenosis in infants and young children. Catheterization and Cardiovascular Interventions. 75(7): 1084-90 (2010). 